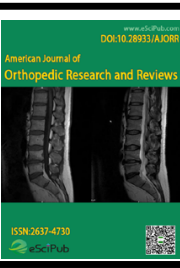

American Journal of Orthopedic Research and Reviews

(ISSN:2637-4730)

\title{
Tibial plateau fractures in a limited-resource setting: A prospective study of surgical techniques and outcome over three years
}

Daniel Handy Eone ${ }^{1^{*}}$, Itambi Maxwell Asoh ${ }^{1}$, Ernest Kikah Ndifor ${ }^{1}$, Mbaga Ntjam Andre Claude ${ }^{1}$, Fonkoue Loic ${ }^{2}$, Muluem Olivier Kennedy ${ }^{1}$, Guifo Marc Leroy ${ }^{3}$, Kaldadak Martin ${ }^{4}$, Ibrahima Farikou $^{5}$

${ }^{1}$ Orthopaedic and Trauma Surgery Unit, Yaounde Central Hospital. ${ }^{2}$ Orthopaedic and Trauma Surgery Unit, Yaounde Emergency Centre. ${ }^{3}$ Orthopaedic and Trauma Surgery Unit, Yaounde University Teaching Hospital. ${ }^{4}$ Orthopaedic and Trauma Surgery Unit, Yaounde Military Hospital. ${ }^{5}$ Department of Surgery and Specialties, Faculty of Medicine and Biomedical Sciences, The University of Yaounde I.

\section{ABSTRACT}

Introduction: Tibial plateau fractures form a wide spectrum of injuries accounting for $1.2 \%$ of all fractures and a prevalence of 10 cases per 100,000 inhabitants. Methodology: A prospective consecutive multicentre study from May 2018 to May 2021 was carried out in Yaounde. All consenting cases of tibial plateau fracture underwent surgical treatment while patients with pathologic fractures, previous knee osteoarthritis, medically unfit for surgery, and discharging against medical advice were excluded. Data was analysed with SPSS 26.0 and the level of significance set at $p<0.05$. Results:Eighty-four (84) cases of tibial plateau fractures were sampled and 68 consented to surgery. The mean age was $42 \pm 13.6$ years and sex ratio 2.4. Estimated prevalence was 2.2 cases per 100,000 inhabitants. Schatzker type II fractures were most represented (33.3\%). The left leg was affected in $57.1 \%$. Motorbike accidents were the main cause of injury (66.7\%). Of the 68 operated, $63.3 \%$ by plating osteosynthesis, $32.4 \%$ by external fixation, and $4.4 \%$ by screws fixation. Tricortical iliac bone graft was realised in 4 cases. The minimum follow-up was 6 months, with a median of 18 months (5 to 37 months). Plating osteosynthesis ( $p<0.001$ ), operative time between 60 to 120 minutes $(p<0.02)$ and a good radiologic fracture healing $(p<0.04)$ were associated with a satisfactory outcome. Poor prognosis was seen with open fractures $(p<0.001)$, bridging external fixation $(p<0.001)$, and Schatzker VI fractures $(p<0.02)$. Complications included post-traumatic osteoarthritis (64.7\%), post-traumatic osteomyelitis (29.4\%), knee ankylosis (5.9\%), and limb malalignment (30.9\%). Conclusion:The prevalence of tibial plateau fractures remains lower than reported in literature but it is projected to rise. Plating remains a viable treatment option. A larger scale study will establish the burden of this entity in our context.

Keywords: Tibial plateau fracture, Treatment, Outcome, Complications
*Correspondence to Author:

Daniel Handy Eone

Tel: 00237694743801.

How to cite this article:

Daniel Handy Eone, Itambi Maxwell Asoh, Ernest Kikah Ndifor, Mbaga Ntjam Andre Claude, Fonkoue Loic, Muluem Olivier Kennedy, Guifo Marc Leroy, Kaldadak Martin, Ibrahima Farikou. Tibial plateau fractures in a limited-resource setting: A prospective study of surgical techniques and outcome over three years. American Journal of Orthopedic Research and Review,2022, $5: 31$.

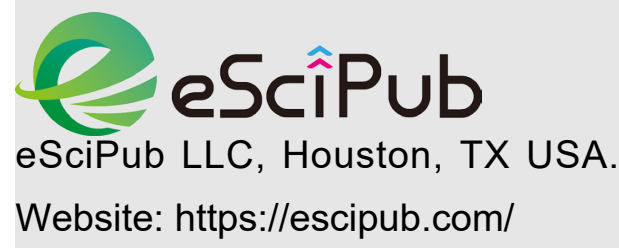




\section{Introduction}

Tibial plateau fractures form a wide spectrum of injuries of the proximal tibia presenting varying challenges with a prognosis that is largely dependent on the management of each particular configuration. General treatment guidelines for these intra-articular fractures are clear; nonetheless, complication rates in the short- to long-term remain relatively high [1]. Knee ankylosis, infection, post-traumatic osteoarthritis, malunion, and nonunion account for most of the complications described; often requiring reinterventions with all its associated burden on the affected ${ }^{[2]}$.

These fracture patterns are relatively rare accounting for approximately $1.2 \%$ of all fractures, with an incidence of 10 per 100.000 annually. This incidence is noted to be increasing in females above 50 years and decreasing in men above 50 years. Overall, the highest incidence occurs between the ages of 40 and 60 . Associated risk factors include high energy trauma in younger, fitter males and osteoporosis in the elderly female. ${ }^{[1,3-6]}$. Fibular fracture often accompanies tibial plateau injuries ${ }^{[7]}$.

The literature on this subject is quite rich but the management of this entity remains challenging, more so in resource-limited settings considering the belief in and practice of alternative treatment of fractures ${ }^{[4,8]}$. With the increasing use of twowheeled vehicles and an increasing average life expectancy at birth for Cameroonians, the incidence of this pathology and its attendant effects can be projected to rise ${ }^{[8-10]}$. A few retrospective studies in our setting have described the epidemiology and the therapeutic indications ${ }^{[8]}$. We, therefore, carried out a prospective cohort study on the surgical management techniques and short-term outcome pertaining to the various fracture patterns seen in our setting.

\section{Methodology}

\section{Study population}

Ethical clearance was obtained from the institutional review board (IRB) of the Faculty of Medicine and Biomedical Sciences of the University of Yaounde I and the review boards of the various study sites. We carried out a prospective study from May 2018 to May 2021 in five Level 2 trauma centres in Yaounde on all patients with tibial plateau fractures. These patients were recruited upon admission in the emergency departments of five level 2 trauma centres in Yaounde. Consenting cases underwent surgical treatment. Pathologic fractures, patients unfit for surgery, history of knee osteoarthritis, and discharge against medical advice were excluded from the study.

\section{Study procedure and database}

Our study was carried out in three phases: preoperative, perioperative, and postoperative phases.

In the preoperative phases, we started by identifying the study sites and the five chosen accounted for the management of at least $95 \%$ of tibial plateau injuries in the city of Yaounde. Then a pretested data entry sheet was used to recruit all tibial plateau fracture cases. It considered information on the socio-demographics (age, sex, level of education), mechanism of injury, the imaging modality which permitted fracture classification using the Schatzker model, and associated lesions. Patients consenting to surgical care were then administered the rest of the questionnaire.

The perioperative phase noted the type of anaesthesia, surgical approach, operative findings, fixation method, any supplementary gestures, blood loss, and the duration of the procedure. Various procedures were adopted for different fracture patterns. Closed fractures with satisfactory soft tissue, according to Tscherne classification, were managed by open reduction and internal fixation (ORIF) with or without fluoroscopic guidance; with the patient in supine position. For lateral condyle fractures, a bump was placed under the ipsilateral gluteal region; and for medial condyle fractures, it was placed under the contralateral gluteal area. Surgical exposures were either anterolateral for lateral condyle patterns; medial or posteromedial for medial patterns; and either combined or midline for complex patterns (Schatzker V, VI). Percutaneous approaches were generally adopted for open fractures or fractures with poor cutaneous covering. 
Fixation methods ranged from unilateral buttress plating for isolated condylar fractures through dual plating in complex patterns to external fixation which was either knee-spanning or a hybrid construct. Autologous tricortical iliac bone graft was realised in some cases.

Open procedures were closed up under suction drains that were generally removed by the third post-operative day (POD). Blood loss and duration of surgery were considered as cumulative for all procedures carried out on the same patient under the same anaesthesia time.

Upon completion of the procedure, the patients were conducted directly to the radiology suite for control radiographs (anteroposterior and lateral views). These permitted appreciation of fracture reduction which was considered as perfect $(<2$ $\mathrm{mm})$, acceptable $(2-3 \mathrm{~mm})$, or poor (>4 mm). Perfect and acceptable reductions were designated satisfactory and a poor reduction considered unsatisfactory. Passive rehabilitation exercises were commenced on POD 1. Partial weight-bearing was allowed from 6 weeks after surgery and full weight bearing at confirmed fracture union (at least 12 weeks).

The postoperative phase assessed the clinical, radiological, and functional outcome according to the follow-up calendar of the attending surgeon (2 weeks, 6 weeks, 10 weeks, 3 months, and at 6 months).

The clinical assessment noted limb alignment and concluded on either a neutral, varus, or valgus alignment. The amplitude of knee joint movement was measured with a goniometer and reported in degrees. This ranged from complete extension at $0^{\circ}$ to complete flexion at $120^{\circ}$. Any reinterventions were noted and their various indications highlighted. Diagnosed cases of posttraumatic osteoarthritis (PTOA) were further assessed radiologically and classified according to the Ahlbäck classification.

Radiological assessment focused on the quality of fracture union on anteroposterior and lateral radiographs. This was evaluated using the standard Radiographic Union Scale in Tibia fractures (RUST) (Table I). This assessment was carried out by an independent review of radiographs by the principal investigator and a senior orthopaedic surgeon to determine fracture union. This was then reported on a scale from 4 to 12. Each cortex was scored as: $1=$ no callus, 2 $=$ callus present, $3=$ remodelled, fracture not visible. Union in at least 3 cortices was considered satisfactory.

Table I: Radiologic Union Scale in Tibial fractures

\begin{tabular}{lll}
\hline & Radiographic Criteria & \\
\hline Score per cortex & Callus & Fracture line \\
1 & Absent & Visible \\
2 & Present & Visible \\
3 & Present & Invisible \\
\hline
\end{tabular}

Table II: Fixation methods

\begin{tabular}{lcc}
\hline Fixation method & $\begin{array}{c}\text { Frequency } \\
(\mathrm{N}=68)\end{array}$ & Percentage \\
\hline Screws only & 3 & 4.4 \\
Buttress plating and graft & 4 & 5.9 \\
Buttress plating only & 35 & 51.5 \\
Double plating only & 4 & 5.9 \\
Monoplanar external fixation & 2 & 2.9 \\
Double planar external fixation & 1 & 1.5 \\
Hybrid external fixation & 8 & 11.8 \\
Bridging external fixation & 8 & 11.8 \\
External fixation and screws & 3 & 4.4 \\
\hline
\end{tabular}


Functional outcome was measured and reported according to the tenets of the International Knee Documentation Committee subjective evaluation form (IKDC). This score was presented as: excellent (80 - 100), good (70 to 79 ), fair (50 to 69), and poor $(<50)$.

\section{Data management}

Data from the study questionnaire was entered and reorganized on CSPro 7.5 from where it was exported to SPSS version 26.0 for analysis. Results were presented as mean, median, and standard deviations for continuous variables and frequencies and percentages used for categorical data. Associations were established using relative risk with level of significance fixed at $p<0.05$ for a confidence interval at $95 \%$. Bivariate analyses were used to deduce the factors affecting the outcome.

\section{Results}

From May 2018 to May 2021, we sampled 84 cases of tibial plateau fractures of whom 68 underwent surgical treatment. The population was male dominated with a sex ratio of 2.4. Ages ranged from 20 years to 70 years with a mean of $42.04 \pm 13.62$ years. Fracture prevalence was estimated at 2.2 cases per 100,000 inhabitants. Schatzker type II fractures were the most represented pattern at $33.3 \%$. Cumulatively, motorbikes accounted for $56(66.7 \%)$ of the injuries followed by motor vehicle injuries and falls (Figure 1). Ipsilateral femoral fracture, pelvic injury, and traumatic brain injury were the most associated lesions in $8(28.6 \%), 5(17.9 \%)$, and $4(14.3 \%)$ of the cases respectively. The left leg was affected in $57.1 \%$ and open fractures seen in $23.8 \%$.

Of the 68 patients operated, plating osteosynthesis was the predominant technique as seen in [43(67.3\%)] followed by external fixation [22(32.4\%)]. Screws were used in isolation in $3(4.4 \%)$ cases (Table II). Autologous tricortical iliac bone graft was realised in 4 cases without any donor site morbidity reported. Anterolateral approach was employed in $41(60.3 \%)$ cases while the posteromedial approach was carried out in $5(7.3 \%)$ cases. Percutaneous approach [22(33.8\%)] was mainly seen in external fixation procedures. Supplementary gestures included meniscectomy, other fracture fixation, spinal stabilization, facial reconstruction and medial gastrocnemius rotationplasty for type IIIB open fractures. Spinal anaesthesia was most common (80.9\%).

Hardware removal for fracture healing and posttraumatic osteomyelitis were the most common indications for re-operation accounting for $19(54.3 \%)$ and $12(34.3 \%)$ respectively. Varus deformity was the most common axial deformity as seen in $18(26.5 \%)$ of cases. Fracture reduction was satisfactory in $75 \%$ of cases. Radiological outcome was marked by fracture healing in $61(89.7 \%)$ cases and $42(61.8 \%)$ who presented with grade 1 posttraumatic osteoarthritis (PTOA). The functional outcome was generally satisfactory with an average IKDC of $75.93 \pm$ 21.25 .

Factors associated with a satisfactory functional score included buttress plating $(p<0.001)$, an operative time between 60 to 120 minutes $(p<0.02)$, and a good radiologic fracture healing $(p<0.04)($

Table III). Factors associated with an unsatisfactory functional result included: open fractures $(p<0.001)$, bridging external fixation $(p<0.001)$, and Schatzker VI fractures $(p<0.02)$ (

\section{Table III).}

\section{Discussion \\ Demographics}

Most of the nonoperated opted for alternative form of fracture treatment. It is still a common belief and practice in many communities in our setting that fractures can be managed by traditional bone setters ${ }^{[8,11]}$.

Bizole et al., deduced that patients sought hospital care depending on their perception of the gravity of their injury, their belief in the efficacy of the health system, and the proximity to a health facility [12].

The yearly trend in fracture incidence revealed a rising curve that peaked in 2019 (32.4\%) and gradually fell to the lowest value in 4 years in 2021 (11.8\%). The estimated prevalence for tibial plateau fractures in over the study period of 
2.2 per 100,000 inhabitants annually was much lower than the 10.3 per 100,000 reported by Elsoe et al., probably due to the inexhaustive inclusion of treatment sites ${ }^{[3]}$.

The mean age of $42.04 \pm 13.62$ years reflected the majority youthful population below 50 years $(66.6 \%)$ that characterised our study population. More so, the $70.2 \%$ male population which was mostly involved in commercial motorbike riding presented mainly high energy fracture patterns (Schatzker IV, V, VI). This was also similar to literature findings ${ }^{[3,13]}$. In addition to motorbike injuries, we had motor vehicles and falls and this corroborated the studies of Bizole et al., Handy et al., and others $[3,11,12,14]$.
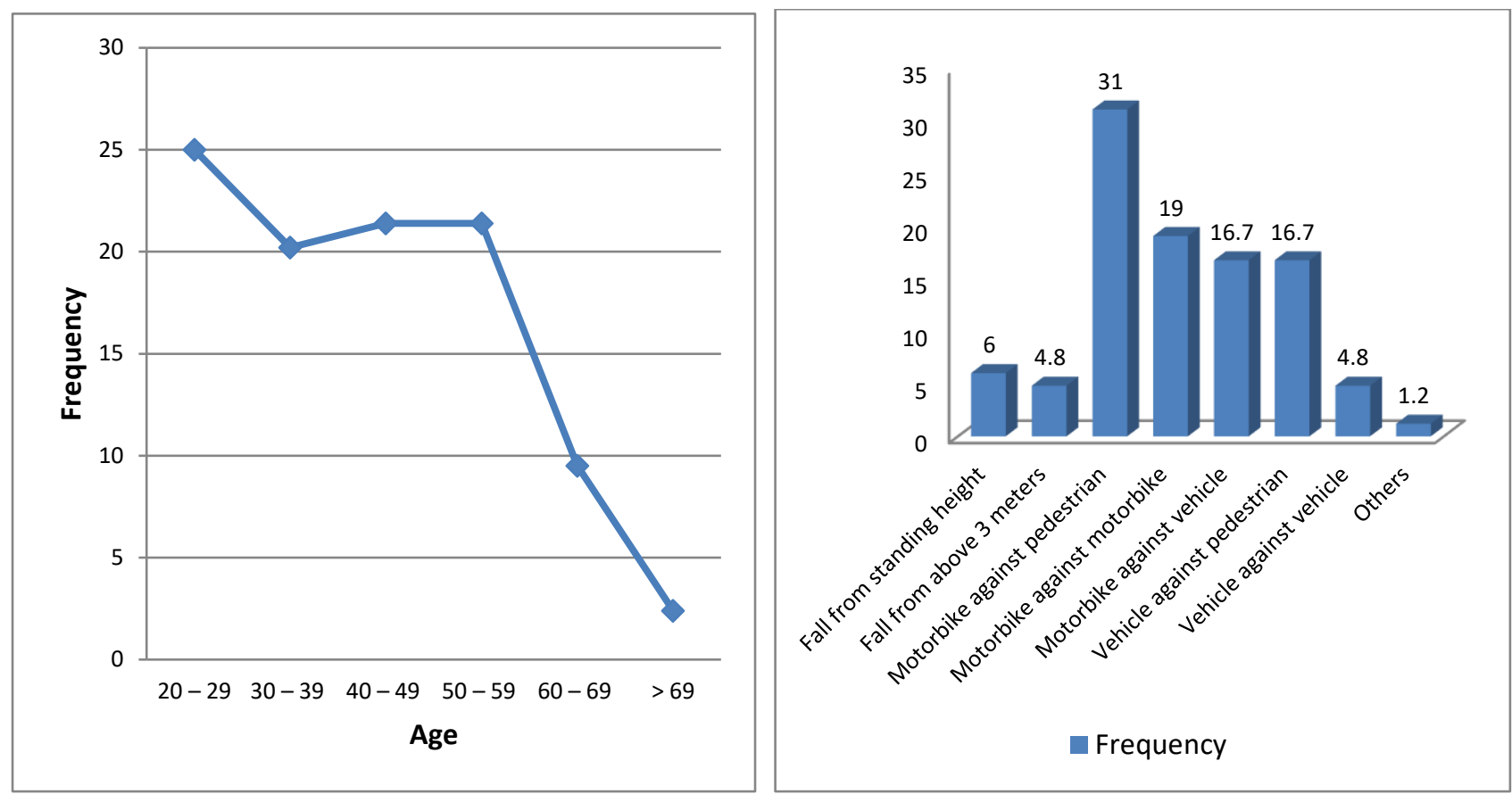

Figure 1: Age distribution and the various mechanisms of injury

\section{Injury characteristics}

The fracture patterns most encountered in our study included: Schatzker type I (8.3\%), II (33.3\%), III (7.1\%), IV (20.2\%), V (16.7\%), and VI (14.2\%). Type II fractures were most represented followed closely by the high energy patterns. This was similar to the $35.1 \%$ and $31 \%$ reported by Albuquerque et al., and Handy et al., respectively [11,13]. The lateral tibial condyle is known to be less dense compared to the medial
(142 $\mathrm{kg} / \mathrm{cm} 3$ versus $236 \mathrm{~kg} / \mathrm{cm} 3$ respectively). Thus it is easily fractured [11].

The morbidity associated with these fracture patterns was compounded by the fact that $33.3 \%$ of the study population had associated lesions dominated by other fractures and traumatic brain injury. Of these patients, $57.1 \%$ were left-sided and $42.9 \%$ right-sided. This is the same as the study by Elsoe et al., in Denmark and Albuquerque et al., in Brazil [3,13].

Table III: Distribution of factors associated with fracture treatment outcome

\begin{tabular}{|l|l|c|c|c|c|c|}
\hline \multicolumn{2}{|c|}{ Variable } & \multicolumn{2}{|c|}{ IKDC scale } & $\begin{array}{c}\text { Relative } \\
\text { Risk (RR) }\end{array}$ & 95\% Cl & $p$-value \\
\cline { 3 - 5 } & & $\begin{array}{c}\text { Satisfactory } \\
(\%)\end{array}$ & $\begin{array}{c}\text { Unsatisfactory } \\
(\%)\end{array}$ & & \\
\cline { 1 - 5 } & Age & $20(67.5)$ & $12(37.5)$ & 1.32 & $0.85-2.05$ & 0.20 \\
\cline { 2 - 5 } & $>40$ years & $17(47.2)$ & $19(52.8)$ & & & \\
\hline
\end{tabular}


Daniel Handy Eone et al., AJORR, 2022, 5:31

\begin{tabular}{|c|c|c|c|c|c|c|}
\hline \multirow[t]{2}{*}{ Skin integrity } & Open & $1((5.9)$ & $16(94.1)$ & 0.08 & $0.01-0.56$ & \multirow[t]{2}{*}{$<0.001^{*}$} \\
\hline & Closed & $36(70.6)$ & $15(29.4)$ & & & \\
\hline \multirow{2}{*}{$\begin{array}{l}\text { Timing from in- } \\
\text { jury to surgery } \\
\text { (days) }\end{array}$} & $<7$ days & $34(57.6)$ & $25(42.4)$ & 1.73 & $0.69-4.47$ & \multirow[t]{2}{*}{0.28} \\
\hline & $7-21$ & $3(33.3)$ & $6(66.7)$ & & & \\
\hline \multirow{4}{*}{$\begin{array}{l}\text { Duration of pro- } \\
\text { cedure } \\
\text { (minutes) }\end{array}$} & $<60$ mins & $1(25)$ & $3(75)$ & 0.44 & $0.02-1.78$ & \multirow[t]{2}{*}{0.32} \\
\hline & $60-120$ & $27(65.9)$ & $14(34.1)$ & 1.78 & 1.04-3.05 & \\
\hline & $>120$ & $9(39.1)$ & $14(60.9)$ & 0.63 & $0.36-1.10$ & \multirow[t]{2}{*}{$0.02^{*}$} \\
\hline & & & & & & \\
\hline \multirow{2}{*}{$\begin{array}{l}\text { Radiological } \\
\text { score }\end{array}$} & Good & $36(59)$ & $25(41)$ & 0.48 & $0.31-0.73$ & \multirow[t]{2}{*}{$0.04^{\star}$} \\
\hline & Poor & $1(14.3)$ & $6(85.7)$ & & & \\
\hline
\end{tabular}

Fixation methods

\begin{tabular}{|c|c|c|c|c|c|}
\hline Screws only & $2(66.7)$ & $1(33.3)$ & 1.24 & $0.54-2.84$ & 1 \\
\hline Buttress plating and graft & $2(50)$ & $2(50)$ & 0.91 & $0.32-2.50$ & 1 \\
\hline Buttress plating only & $29(82.9)$ & $6(17.1)$ & 3.42 & $1.84-6.36$ & $<0.001^{\star}$ \\
\hline Double plating only & $2(50)$ & $2(50)$ & 0.91 & $0.32-2.50$ & 1 \\
\hline Monoplanar external fixation & $0(0)$ & $2(100)$ & 2.28 & $1.73-2.99$ & 0.20 \\
\hline Double planar external fixation & $0(0)$ & $1(100)$ & 2.23 & $1.71-2.91$ & 0.46 \\
\hline Hybrid external fixation & $2(25)$ & $6(75)$ & 0.43 & $0.13-1.45$ & 0.13 \\
\hline Bridging external fixation & $0(0)$ & $8(100)$ & 2.61 & $1.89-3.60$ & $0.001^{*}$ \\
\hline External fixation and screws & $0(0)$ & $3(100)$ & 2.32 & $1.76-3.07$ & 0.09 \\
\hline
\end{tabular}

Schatzker fracture types

\begin{tabular}{|l|c|c|c|c|c|}
\hline I & $3(75)$ & $1(25)$ & 1.41 & $0.77-2.60$ & 0.62 \\
\hline II & $12(57.1)$ & $9(42.9)$ & 1.07 & $0.68-1.70$ & 0.76 \\
\hline III & $4(100)$ & $0(0)$ & 1.94 & $1.53-2.46$ & 0.12 \\
\hline IV & $10(71.4)$ & $4(28.6)$ & 1.43 & $0.93-2.19$ & 0.15 \\
\hline V & $5(38.5)$ & $8(61.5)$ & 0.66 & $0.32-1.32$ & 0.20 \\
\hline VI & $3(25)$ & $9(75)$ & $\mathbf{1 . 9 0}$ & $\mathbf{1 . 2 0 - 3 . 0 2}$ & $\mathbf{0 . 0 2}$ \\
\hline
\end{tabular}

* statistically significant

\section{Treatment}

Fracture assessment was solely with plain radiography. Considering the absence of a health system in Cameroon that facilitates access to fracture care in terms of diagnosis using finer modalities like CT scan, plain radiography was adopted and backed by the ground-breaking works of Schatzker et al., ${ }^{[15]}$. There is yet to be a consensus on the best method of fixation or the best method of addressing bone defects during tibial plateau surgery ${ }^{[16]}$. The treatment methods employed included open reduction and internal fixation by buttress plating associated with autologous tricortical iliac crest graft; hybrid external fixation (Full extension without pain at 12 weeks after surgery and at removal of plaster cast.

Full flexion at 16 weeks after surgery); internal fixation with screws neutralized with external fixation; and spanning knee external fixation. Various plating models were practiced including single or double plating in $67.3 \%$ with a general outcome 
that was satisfactory with regards to fracture reduction and joint congruence after surgery irrespective of whether fluoroscopy was used or not.

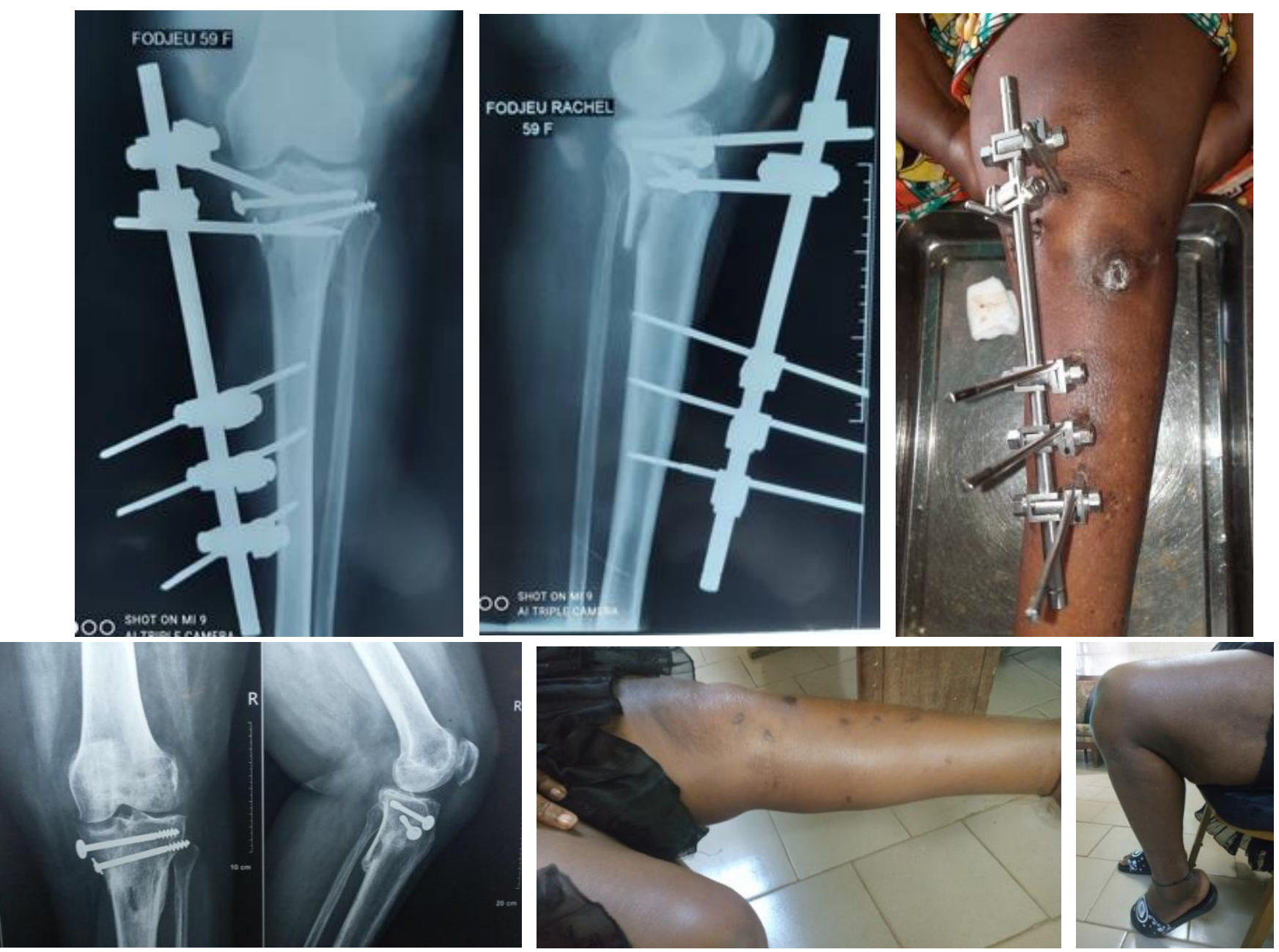

Figure 2: Case of a type I open Schatzker VI fracture treated by joint-sparing ('hybrid') external fixation and screws.

A. Immediate antero-posterior post-operative of open Gustilo 1 Schatzker VI left tibial plateau fracture

B. External oblique view post-operative radiograph showing a posterior fragment that was lagged with 2 cancellous screws under fluoroscopy.

C. Clinical outlook at operative day 7.

D. Removal of external fixator at 8 weeks and placement of a walking plaster boot. Note presence of cancellous screws and fracture consolidation

E. Full extension without pain at 12 weeks after surgery and at removal of plaster cast.

F. Full flexion at 16 weeks after surgery

Even though it was not realised in our study, immediate arthroscopy provides accurate information for fracture reduction, leading to immediate treatment of concomitant intra-articular lesions without complications ${ }^{[17]}$. The posteromedial approach was practiced in $7.3 \%$ of cases especially in medial condylar fractures even though it presented the best approach to fracture fixation compared to the anteromedial approach. Autologous tricortical iliac bone graft was realised in four cases of unicondylar fractures with satisfactory outcome. Alternative means of replacing bone loss in tibial plateau surgery include the use of heterologous bone graft or bone cement. There is still insufficient evidence on the best option, but autologous graft which is probably gold standard in young patients, is associated with severe donor site pain. This has prompted some preference for heterologous grafts and bone cement ${ }^{[18]}$.

With external fixation, hybrid fixation realised with Hoffman frame yielded the best results 
although not statistically significant. In a Cochrane review that compared the use of hybrid fixation versus standard ORIF in people with open or closed Schatzker types V or VI tibial plateau fractures, the function scores tended to favour hybrid fixation in terms of lower risk for an unplanned reoperation, higher mean knee range of motion values, and a higher likelihood of return to pre-injury activity level ${ }^{[16]}$. On the other hand, spanning knee external fixation yielded the worst results.

The treatment of other concurrent fractures was carried out in $20.6 \%$ of the population. This was followed by medial gastrocnemius rotationplasty employed in five cases (7.4\%) for Gustilo type IIIB open fractures. Success was encountered in three cases while two underwent partial necrosis.

The average duration of surgery was 131.7 minutes (range $65-360$ minutes). Shorter operative times were recorded with percutaneous procedures such as external fixation while prolonged operative times were seen in complicated fracture patterns and tibial plateau fractures treated at the same time with associated lesions. But then it was shown to be statistically significant and associated with satisfactory outcome when procedures lasted between 60 and 120 minutes $(p<0.02)$. This could seem to establish the merits of reduced complication rates such as infection when surgical procedures last less than 120 minutes.

\section{Outcome}

All patients were followed up for at least 6 months; with a median follow-up of 18 months (range 6 to 37 months). The functional outcome was satisfactory with a mean IKDC of $75.93 \pm$ 21.25. Our figure represents the general study population with confounding fixations compared to the study by Jae-Jung et al. who scored $85 \pm$ 8 points and $86 \pm 6$ points for ORIF and ORIF with immediate arthroscopy respectively [17]. Kampa et al. reported an average IKDC of 81 (range 55 -97) in arthroscopically-assisted tibial plateau fixations ${ }^{[19]}$. Arthroscopy permits better fracture reduction and concurrent diagnosis and treatment of other intraarticular lesions and thus enhances a better outcome.

The quality of fracture reduction after surgery was satisfactory in $75 \%$ of cases. The tibial plateau is a forgiving joint in terms of minor articular step-off. More so, step-off alone does not account for outcome in terms of post-traumatic osteoarthritis. Other factors include joint stability, retention of the meniscus, and coronal alignment [14].

\section{Conclusion}

We therefore conclude that the management of tibial plateau fractures remains challenging in our setting with an annual incidence that tends to increase. Plating osteosynthesis remains a viable treatment option but hybrid external fixation needs further assessment to establish its validity; considering clinically promising outcomes which was statistically insignificant in this study.

\section{Acknowledgment}

Itambi Maxwell Asoh conceived the research proposal, collected and analysed the data, drafted the article. Daniel Handy Eone, Ernest Kikah Ndifor, Mbaga Ntjam Andre Claude, Fonkoue Loic, Muluem Olivier Kennedy, Guifo Marc Leroy, Kaldadak Martin, and Ibrahima Farikou all contributed to the research proposal, and data analysis. All authors read and approved the final draft for the article.

\section{References}

[1] Mthethwa J, Chikate A. A review of the management of tibial plateau fractures. Musculoskelet Surg. 2018;102(2):119-27.

[2] Papagelopoulos PJ, Partsinevelos AA, Themistocleous GS, Mavrogenis AF, Korres DS, Soucacos PN. Complications after tibia plateau fracture surgery. Injury. 2006;37(6):475-84.

[3] Elsoe R, Larsen P, Nielsen NPH, Swenne J, Rasmussen S, Ostgaard SE. Population-Based Epidemiology of Tibial Plateau Fractures. Orthopedics. 2015;38(9):780-6.

[4] Achor T, Taylor R. Tibial Plateau and Shaft Fractures. Orthopaedic Knowledge Update 12. 12th Edition. USA: AAOS; 2017. p. 494-509.

[5] Marsh J. Tibial Plateau Fractures. Rockwood and Green's Fracture in Adults. Eighth edition. China: Wolters Kluwer; 2015. p. 2814.

[6] van Dreumel RLM, van Wunnik BPW, Janssen L, Simons PCG, Janzing HMJ. Mid- to long-term functional outcome after open reduction and 
internal fixation of tibial plateau fractures. Injury. 2015;46(8):1608-12.

[7] Zheng Z, Yu Y-Y, Chang H-R, Liu H, Zhou H-L. Establishment of Classification of Tibial Plateau Fracture Associated with Proximal Fibular Fracture. Orthop Surg. 2019;11(1):97-101.

[8] Bizolé BY, Essi M, Handy E, Nseme E, Ibrahima F, Sosso M. Orthopedic itineraries and public health challenges in the management of fractures in a tertiary hospital in Yaoundé Cameroon. Int J Orthop Sci. 2019;5(2):674-7.

[9] Cameroon Life Expectancy 1950-2020. Accessed on 29/9/2020.

https://www.macrotrends.net/countries/CMR/cameroon/life-expectancy.

[10] Ngaroua D, Mbo J, Natacha Aidego A, Yaouba D, Ngah J. Les Fractures Dues aux Accidents de la Voie Publique à l'Hôpital Régional de Ngaoundere (Cameroun). Health Sci Dis. 2016;17 (3) :24-8.

[11] Handy ED, Bizole BDY, Essi MJ, Kaldadak K, Ngo Nonga B, Sosso MA. Epidemiological Aspects and Therapeutic Indications of Tibial Plateau Fractures in Adults: About 84 Cases Observed in Yaoundé. Med J Clin Trials Case Stud. 2017;1(3):000118

[12] Bizolé BYD, Essi MJ, Handy ED, Ibrahima F, Sosso MA. Déterminants Épidémiologiques du Recours à la Biomédecine chez les Patients Victimes de Traumatismes Orthopédiques: à
Propos de 214 Cas à Yaoundé. Health Sci Dis. 2019;20(6):69-73.

[13] Albuquerque R, Hara R, Prado J, Schiavo L, Giordano V, Amaral N. Epidemiological study on tibial plateau fractures at a level I trauma center. Acta Ortop Bras. 2013;21:109-15.

[14] Giannoudis PV, Tzioupis C, Papathanassopoulos A, Obakponovwe O, Roberts C. Articular step-off and risk of post-traumatic osteoarthritis. Evidence today. Injury. 2010;41(10):986-95.

[15] Schatzker J, McBroom R, Bruce D. The Tibia1 Plateau Fracture: The Toronto Experience 19681975. Clin Orthop Relat Res. 1979;(138):94104.

[16] McNamara IR, Smith TO, Shepherd KL, Clark $A B$, Nielsen DM, Donell $S$, et al. Surgical fixation methods for tibial plateau fractures. Cochrane Database Syst Rev 2015;(9):CD009679.

[17] Jeong J-J, Oh S, Ji J-H, Park S-J, Ko M-S. Immediate arthroscopy following ORIF for tibial plateau fractures provide early diagnosis and treatment of the combined intra-articular pathologies. Knee Surg Sports Traumatol Arthrosc. 2019;27(10):3327-33.

[18] Prat-Fabregat S, Camacho-Carrasco P. Treatment strategy for tibial plateau fractures: an update. EFORT Open Rev. 2016;1(5):225-32.

[19] Kampa J, Dunlay R, Sikka R, Swiontkowski M. Arthroscopic-Assisted Fixation of Tibial Plateau Fractures: Patient-Reported Postoperative Activity Levels. Orthopedics. 2016;39(3):486-91. 